\title{
Energy loss of monoenergetic positrons passing through a thin carbon foil
}

\author{
B. Yang, C. K. Ng, C. C Ling and S. Fung* \\ Department of Physics, The University of Hong Kong, Pokfulam Road, Hong Kong, People's Republic of China
}

\begin{abstract}
In this paper, the measurements of energy loss and energy loss straggling for 1-10 keV monoenergetic positrons passing through thin carbon foils of different thicknesses ranging from 1.0 to $5.0 \mu \mathrm{g} / \mathrm{cm}^{2}$ are presented. The stopping power $d E / d x$ and positron transmission coefficient as a function of incident positron energy and foil thickness have also been investigated. Particularly, the experimental results obtained are compared with those from Monte Carlo simulation and theory with a view to providing a way to determine the actual thickness of a carbon foil. The ratio of the energy straggling to the foil thickness seems to have a linear relation with the beam energy.
\end{abstract}

* Corresponding author. E-mail address: sfung@hkucc.hku.hk 


\section{Introduction}

Thin carbon foils are very important for many instruments used for time-of flight mass spectrometry [1,2] and energetic neutral atom imaging [3,4]. In our previous proposed positron lifetime beam design [5], the positron transiting the thin carbon foil hence generating secondary electrons is used to produce the start signal for the lifetime measurement (the stop signal coming from the eventual annihilation of the positron). However, the interaction between positrons and target atoms may have adverse effects such as energy loss and straggling that will significantly decrease the resolution of the system. Therefore, a study on the total stopping power of positrons defined as the mean energy loss per unit pathlength and energy straggling is of vital importance.

Positrons transiting a solid lose their energy mainly by nonradiative collision and radiative collision with atomic electrons [6]. Many workers have developed the theory of collision loss for electron and positron $[7,8]$. While the positron stopping power at energies above $10 \mathrm{keV}$ was well described theoretically by Berger and Seltzer [9] and the ICRU Report 37 [10], Batra [11] had given the stopping power formula for positrons and electrons by a two-parameter approximation and the formula seems to be valid in the energy ranging from 1 $\mathrm{keV}$ to $500 \mathrm{keV}$.

This article aims to provide some useful data for better understanding the interaction between positrons and carbon foils and the possible relationship between positron beam parameters and foil thickness.

\section{Experimental}

The experimental setup was based on the slow positron beam facility at the University of Hong Kong [12]. Thin carbon foils with different thickness ranging from 1.0 to $5.0 \mu \mathrm{g} / \mathrm{cm}^{2}$ were mounted on a $15 \mathrm{~mm}$ tungsten mesh with a transmission rate of around $65 \%$ and placed perpendicular to the incidence. Double grids with transmission rate larger than $95 \%$ were placed behind the carbon foil for retarding positrons. A micro channel plate (MCP) for detection was placed after the retarding grids. The positrons impacting on the MCP were detected by using standard electronics. 


\section{Results and discussion}

The positron transmission coefficient is defined to be the ratio of transmitted positrons over incident positron counts and was measured as a function of positron energy for different target thicknesses in Fig. 1. It can be seen that, for all different carbon foils, the transmission is small at low incident positron energy. However, the curves rise quickly and saturate at energies above $6 \mathrm{keV}$. It can also be concluded from these curves that the positron transmission decreases for thicker carbon foil. The inner plot in Fig. 1 shows the simulation results of Monte Carlo simulation program CASINO [13]. CASINO was used to simulate the transmission for electrons. The transmission of electrons saturates to $100 \%$ above $6 \mathrm{keV}$. The saturation point is the same as our result although the saturation value is somewhat larger than our experimental result. The reason for this may be related to some contamination on the foil surface and the uncertainty about the foil thickness which are not taken into account in the simulation.

Fig. 2 presents the energy distribution for a $5 \mathrm{keV}$ positron beam after passing through different foils. From Fig. 2, the shift of the peaks with different foil thickness can be clearly observed. It may be concluded that as the carbon foil thickness increases the transmitted positron energy distribution is broadened by more straggling and exhibits a larger average energy loss.

Here it is simply assumed that the total stopping power is roughly equal to the energy loss $\Delta E$ divided by the foil thickness $t$, i.e.

$$
S=\frac{d E}{d t} \approx \frac{\Delta E}{t}=\frac{E_{B}-E_{l}}{t}
$$

where $E_{B}$ is the energy of incident positrons and $E_{l}$ is the mean energy calculated from the positron energy distribution after the carbon foil. Eq. (1) is considered as a good approximation since the foils are sufficiently thin.

Fig. 3 shows our experimental results for the energy loss of the positrons. The calculated stopping power is present in Fig. 4 and some simulation and theoretical results are also plotted for comparison. CASINO was again used to simulate electron transmission. 
ESTAR calculated the stopping-power tables for electrons according to methods described in the report 37 and 49 of ICRU [14]. Batra [11] gave the formula of stopping power for positrons in carbon foil, i.e.

$$
-\frac{1}{\rho}\left(\frac{d E}{d x}\right)=1.4 \beta^{-1.7016}
$$

where $\rho$ is the density of carbon and $\beta$ is the velocity of incident positrons in units of the velocity of light. It can be seen that ESTAR and CASINO are quite similar and they have a good agreement with Batra's formula above $2 \mathrm{keV}$. There is a large discrepancy between our measurements and theoretical results. This may be due to the incorrect nominal thickness and any possible contamination of the foil surface may also influence the smoothness of the measured curve. Assuming that the stopping power curves for all foils follow the trend of Batra's formula, the actual thicknesses were calculated by dividing the measured results with Batra's results and plotted in Fig. 5. This gives a very good linear fit and similar results can also be found in the work of Allegrini et al [15].

The energy straggling $\mathrm{E}_{\mathrm{FWHM}}$ (defined as the full width at half maximum of the energy loss distribution) was also measured and presented in Fig. 6. Here, the convolution of incident beam profile and the energy straggling due to the target has been taken into account. The upper panel in Fig. 6 shows that the $E_{\text {FwHM }}$ varies as a function of incident positron energy. The lower panel shows the same data except being divided by the corrected foil thickness t'.

Our measurements show that the $E_{\text {FWHM }}$ varies as t', not $\sqrt{t^{\prime}}$ as found by Allegrini et al [14]. Furthermore, $\mathrm{E}_{\mathrm{FWHM}} / \mathrm{t}$ ' follows a linear law in our result, i.e.

$$
\frac{E_{F W H M}}{t^{\prime}}=a E_{B}+b, \text { for } 1<E_{B}<10 \mathrm{keV}
$$

where a and b are constants, instead of the power law in [15].

\section{Conclusion}

The transmission coefficient for positrons passing through carbon foil has been measured. The trend is found to be very similar to the result of CASINO. The discrepancy for the saturation value is most likely due to the contamination on the foil surface and the uncertainty 
about the foil thickness. The energy loss and stopping power were calculated from the transmitted positron energy distributions. The results presented in the paper were also compared with those of simulation and theory.

It was found that CASINO and ESTAR agreed with Batra's formula at incident energy larger than $2 \mathrm{keV}$. On adopting the Batra's form for the stopping power, the actual thicknesses were calculated after comparing the difference between measurements and the results of Batra's. It was finally shown that the ratio of the energy straggling to the corrected thickness seems to have a linear relation with the beam energy.

\section{ACKNOWLEDGEMENTS}

The work described in this paper is supported by the GRF grant from the Research Grant Council of the Hong Kong Special Administrative Region, China (under project no. HKU7021/10P).

\section{References}

[1] M. Wüest, in Measurement Techniques in Space Plamas: Particles, AGU Monograph 102, edited by R. F. Pfaff, J. E. Borovsky, and D. T. Young (American Geophysical Union, Washington DC, 1998), pp. 141-155.

[2] D. J. McComas, F. Allegrini, C. J. Pollock, H. O. Funsten, S. Ritzau, G. Gloeckler, Rev. Sci. Instrum. 75, 4863 (2004).

[3] D. J. McComas, B. L. Barraclough, R. C. Elphic, H. O. Funsten III, M. F. Thomsen, Proc. Natl. Acad. Sci. U.S.A. 88, 9589 (1991).

[4] H. O. Funsten, D. J. McComas, B. L. Barraclough, Opt. Eng. (Bellingham) 32, 3090 (1993).

[5] D. Chen, J. D. Zhang, C. C. Cheng, C. D. Beling, S. Fung, Appl. Surf. Sci. 255, 122 (2008).

[6] P. B. Pal, V. P. Varshney, D. K. Gupta, Nucl. Instrum. Methods, B 16, 1 (1986).

[7] H. A. Bethe, Ann. Phys. 5, 325 (1930).

[8] F. Rohrlich, B. C. Carlson, Phys. Rev. 93, 38 (1953).

[9] M. J. Berger, S. M. Seltzer, Stopping powers and ranges of electrons and positrons, (National Bureau of Standarts Report, NBSIR 82-2550 A, 1982).

[10] ICRU, Report No. 37, 1984, Stopping powers for electrons and positrons. (International Commissionon Radiation Units and Measurements, Bethesda, MD, 1984).

[11] R. K. Batra, Nucl. Instrum. Methods. B 28, 195 (1987).

[12] C. K. Cheung, P. S. Naik, C. D. Beling, S. Fung, H. M. Weng, Appl. Surf. Sci. 252, 3132 (2006). 
[13] CASINO, Monte Carlo Simulation of Electron Trajectory in Solids (Université de Sherbrooke, Sherbrooke, Quebec, Canada) http://www.gel.usherb.ca/casino/ [14] M. J. Berger, J. S. Coursey, M. A. Zucker, J. Chang, ESTAR, PSTAR and ASTAR: Computer Programs for Calculating Stopping-Power and Range Tables for Electrons, Protons and Helium Ions (Version 1.2.3) (Gaithersburg, MD: National Institute of Standards and Technology) available at http://physics.nist.gov/Star (2005)

[15] F. Allegrini, D. J. McComas, D. T. Young, J.-J. Berthelier, J. Covinhes, J.-M. Illiano, J.-F. Riou, H. O. Funsten, R. W. Harper, Rev. Sci. Instrum, 77, 044501 (2006).

\section{Figure Captions:}

Figure 1 Positron transmission coefficient as a function of positron energy on carbon foils with different thicknesses. The inner plot shows the simulation results of CASINO for electron in the $2.0 \mu \mathrm{g} / \mathrm{cm}^{2} \mathrm{C}$-foil.

Figure 2 Energy distribution for $5 \mathrm{keV}$ positron beam after passing through carbon foils with different thicknesses.

Figure 3 Energy loss of positron beam after carbon foils with different thicknesses as a function of incident positron energy.

Figure 4 Calculated stopping power of positrons under different foils as a function of incident positron energy. Some simulation and theoretical results are also plotted.

Figure 5 Plot showing the corrected thickness t' as a function of the nominal value $t$ given by the manufacturer. The red line is the linear fit to the points.

Figure 6 Energy straggling $\mathrm{E}_{\mathrm{FWHM}}$ (upper panel) and energy straggling divided by the corrected foil thickness t' as a function of incident positron energy. 


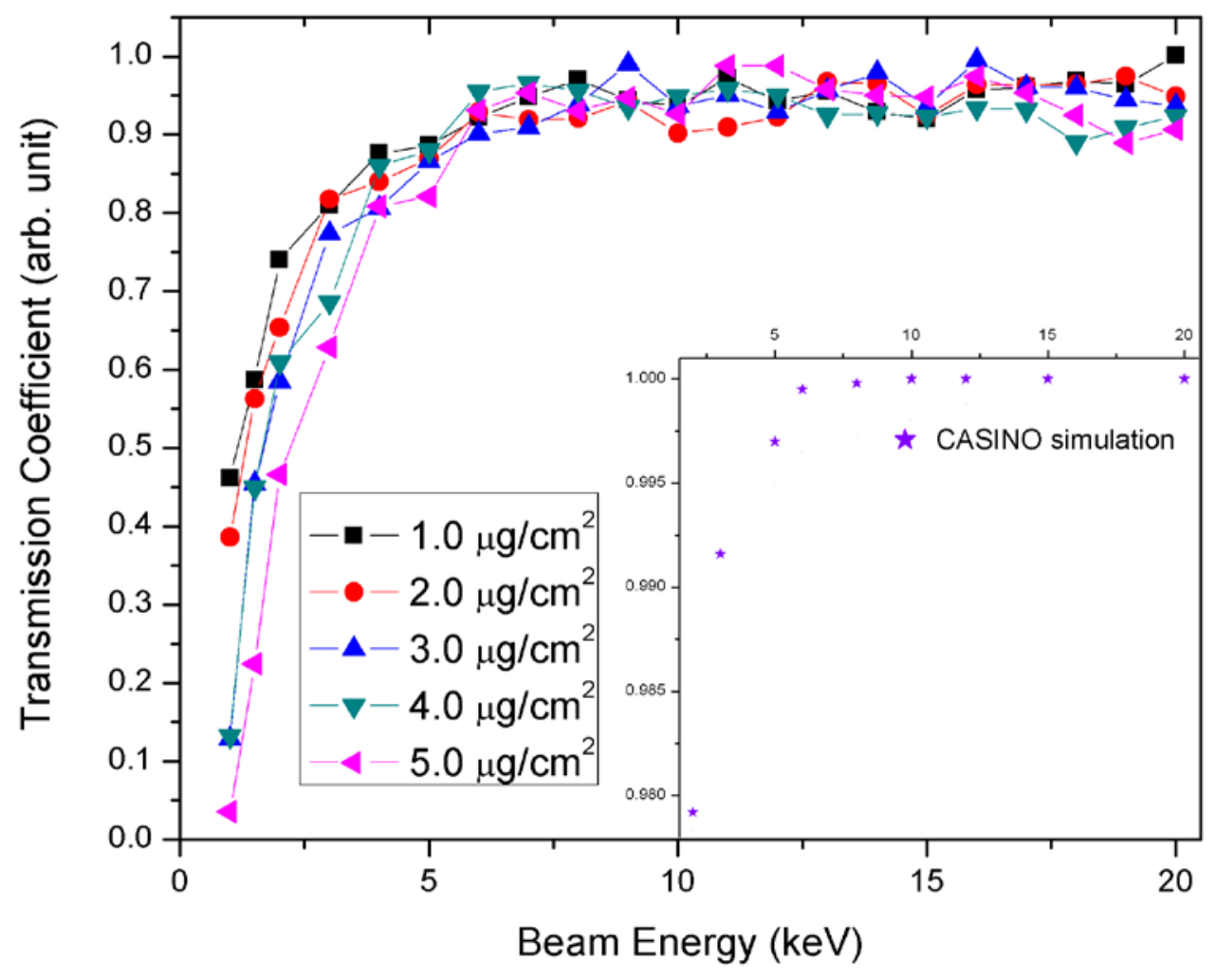

Fig 1 


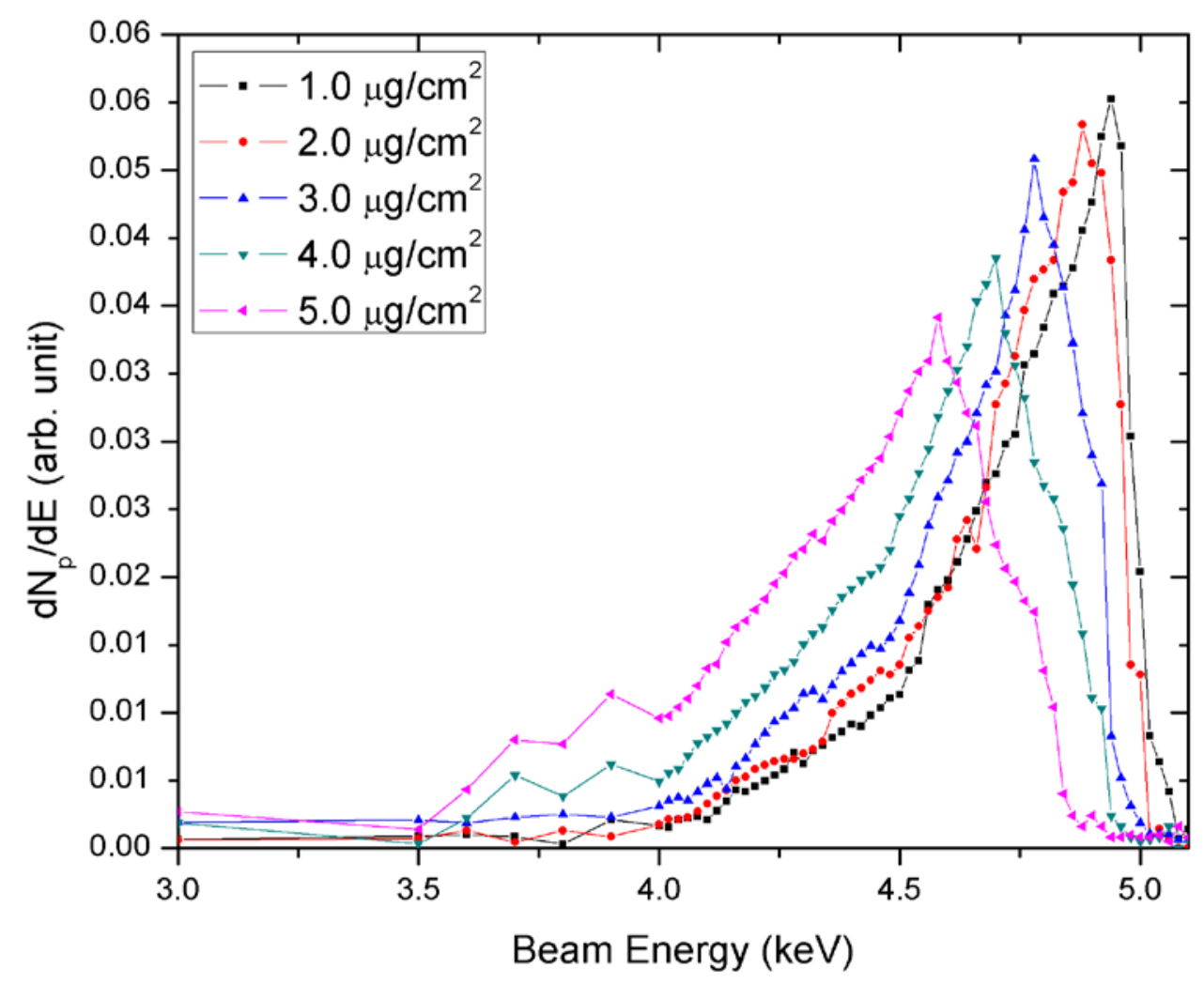

Fig 2 


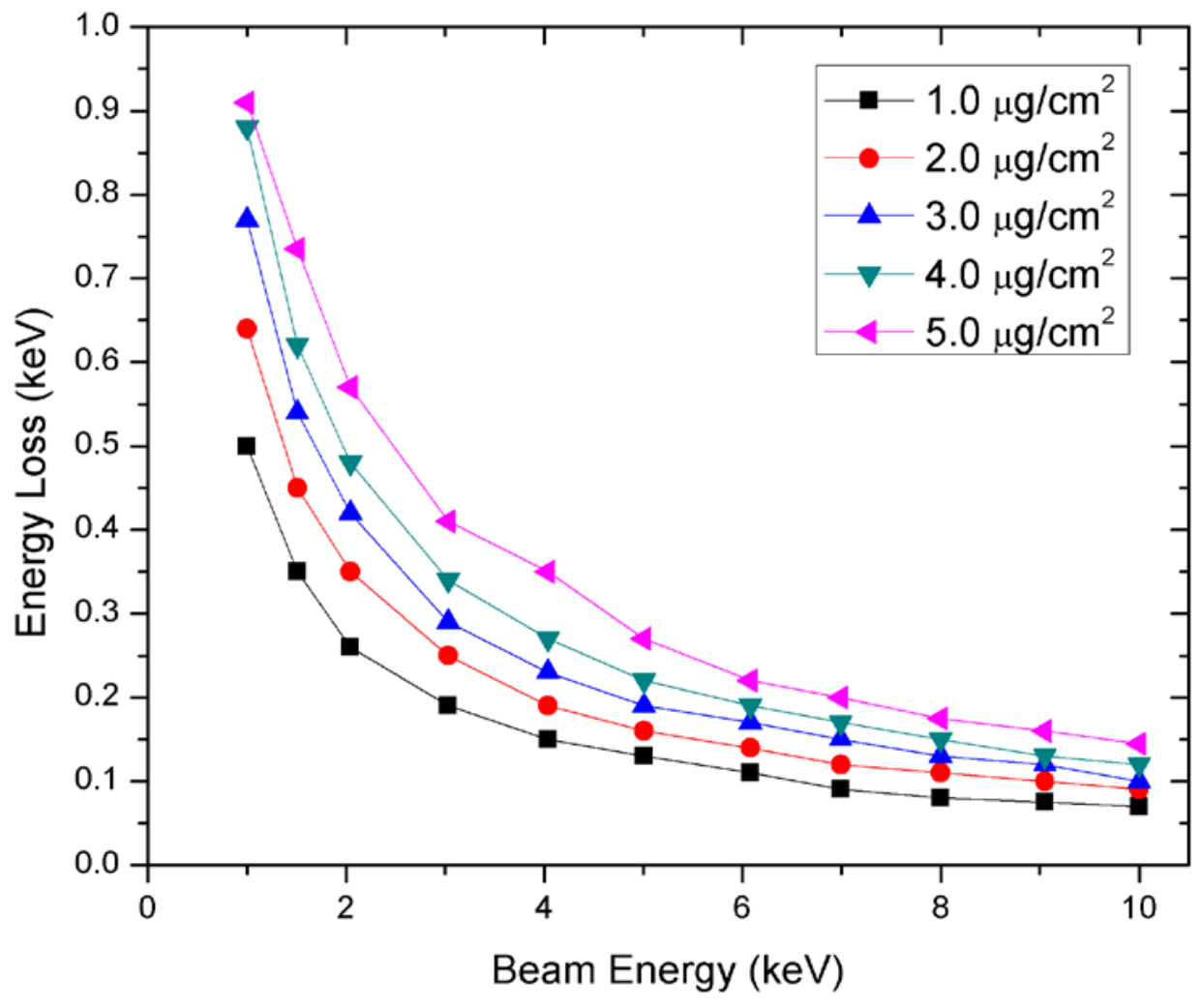

Fig 3 


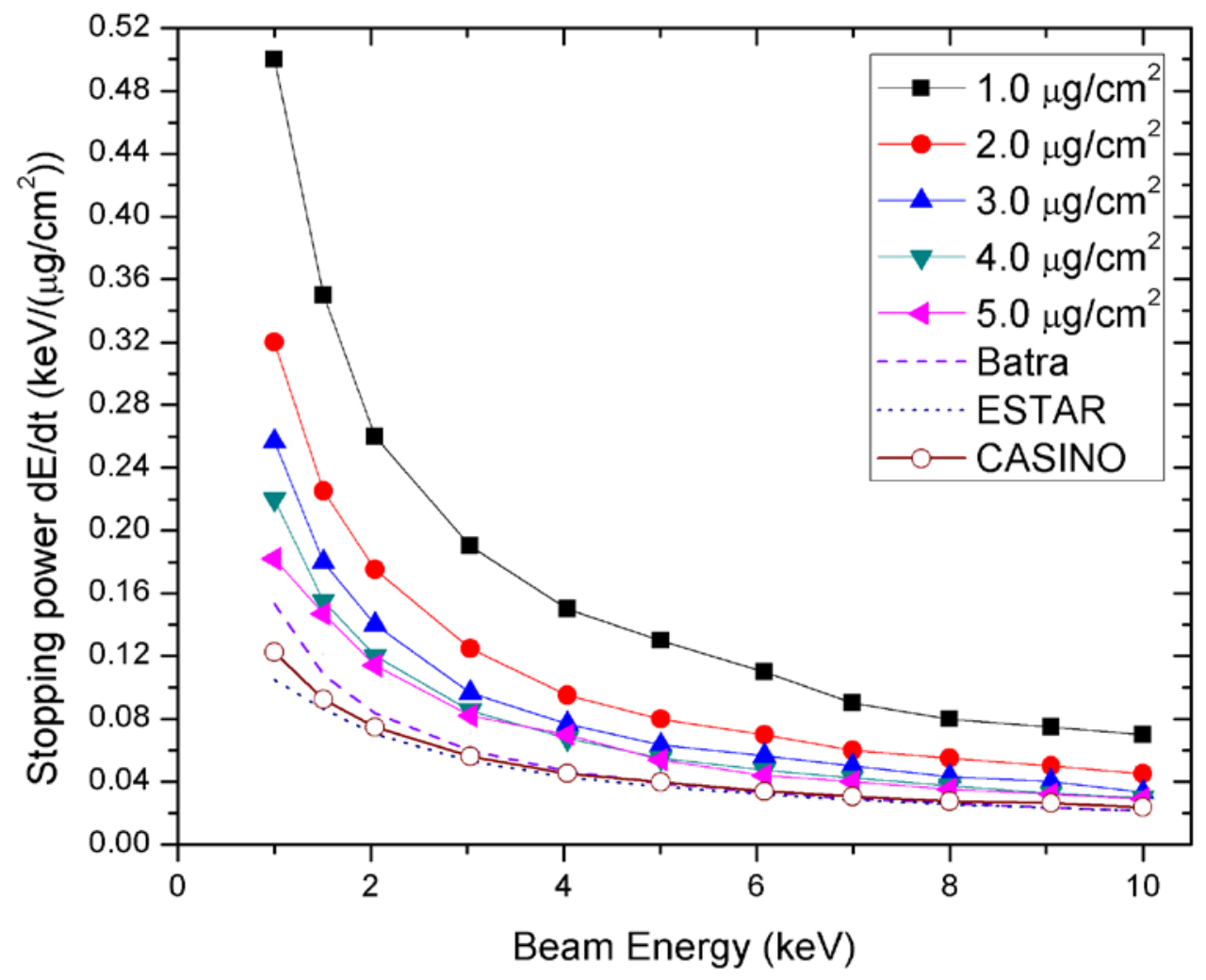

Fig 4 


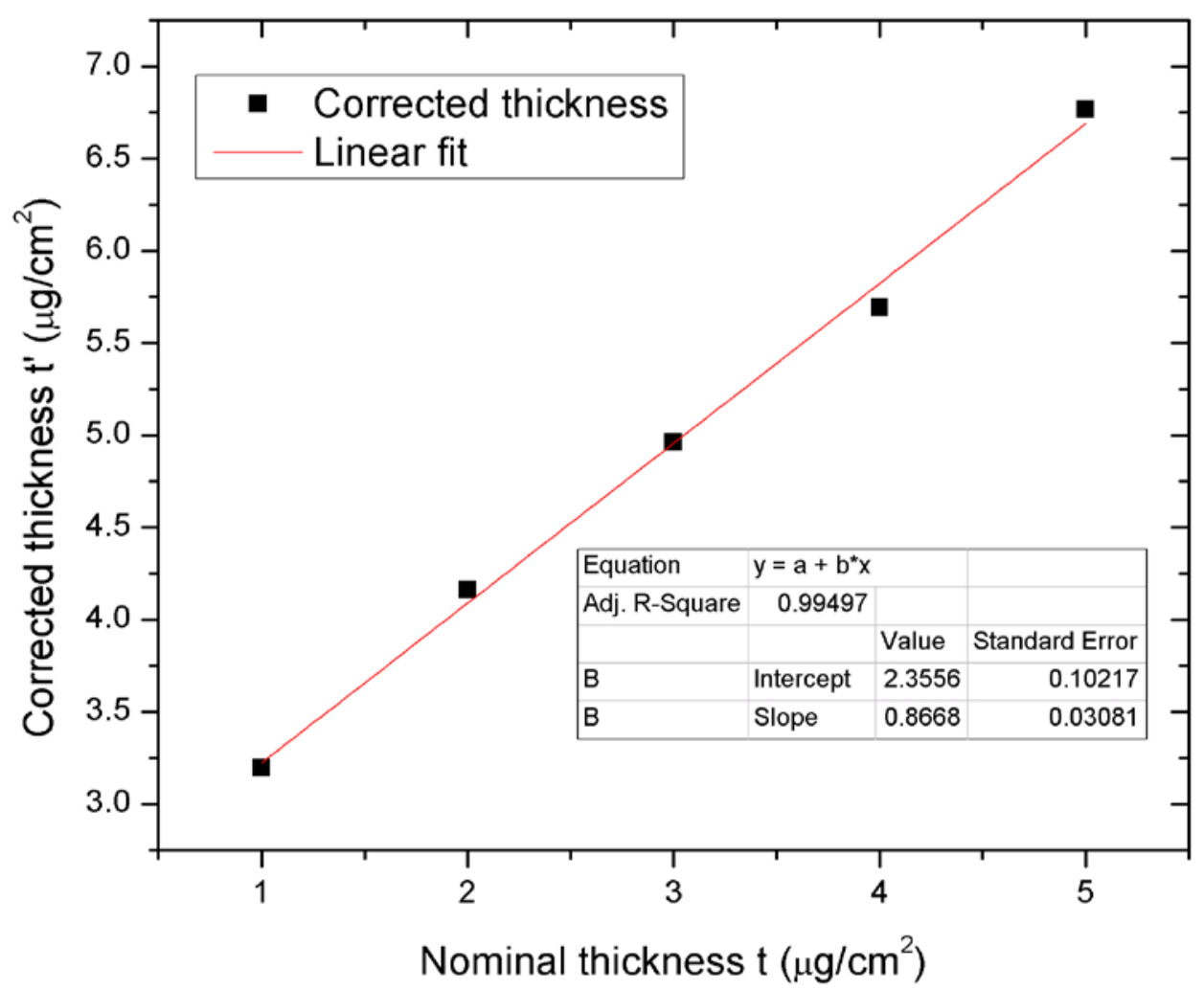

Fig 5 


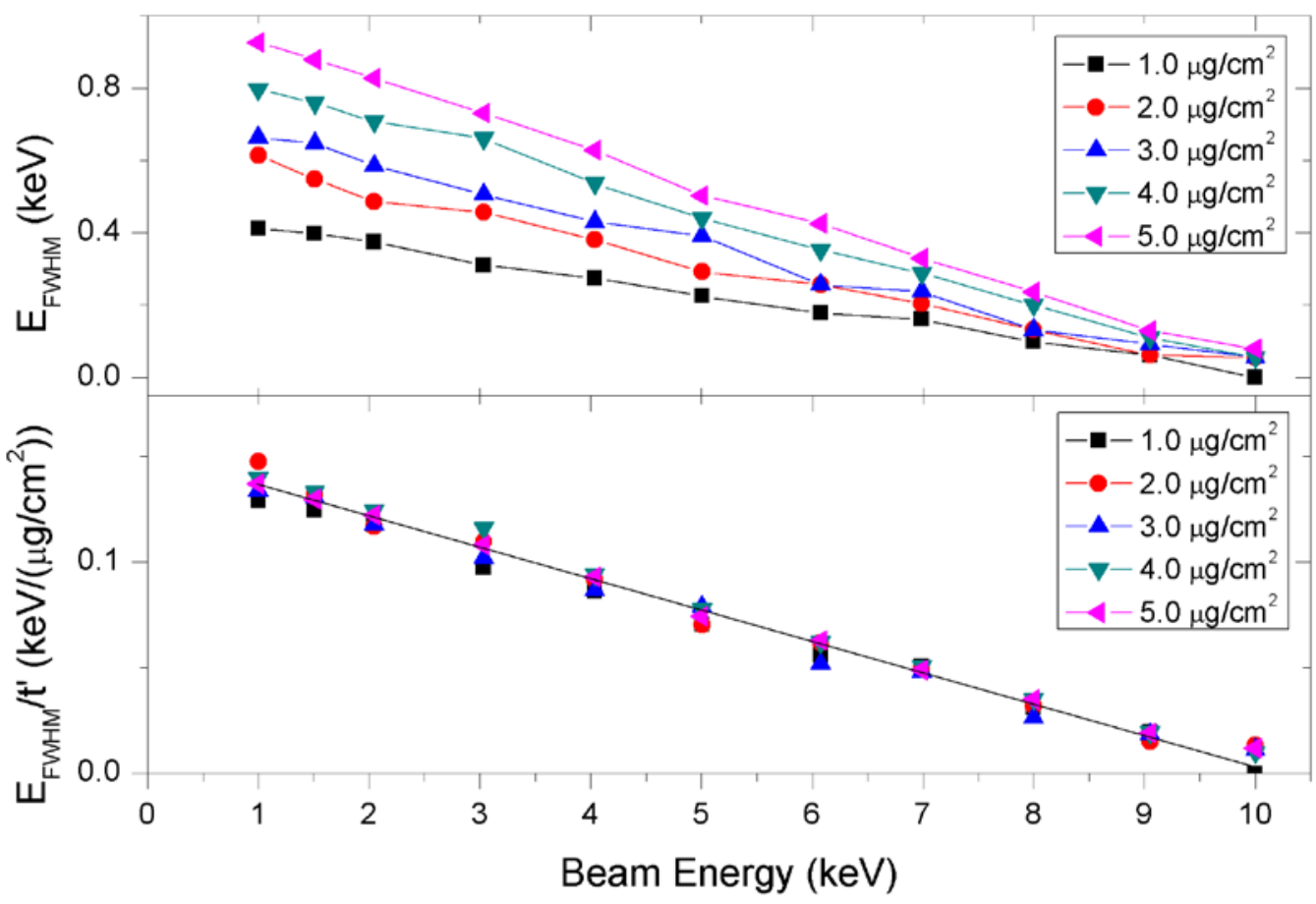

Fig 6 